\title{
A specialised finite element for simulating self-healing quasi-brittle materials
}

\author{
Brubeck L. Freeman ${ }^{1 *}$, , Pedro Bonilla-Villalba ${ }^{1}$, Iulia C. Mihai ${ }^{1}$, Waled F. Alnaas ${ }^{2}$ and Anthony D. Jefferson ${ }^{1}$
}

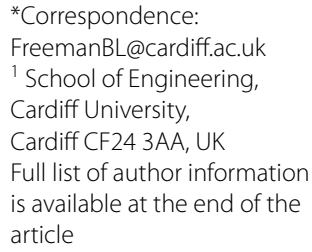

\begin{abstract}
A new specialised finite element for simulating the cracking and healing behaviour of quasi-brittle materials is presented. The element employs a strong discontinuity approach to represent displacement jumps associated with cracks. A particular feature of the work is the introduction of healing into the element formulation. The healing variables are introduced at the element level, which ensures consistency with the internal degrees freedom that represent the crack; namely, the crack opening, crack sliding and rotation. In the present work, the element is combined with a new cohesive zone model to simulate damage-healing behaviour and implemented with a crack tracking algorithm. To demonstrate the performance of the new element and constitutive models, a convergence test and two validation examples are presented that consider the response of a vascular self-healing cementitious material system for three different specimens. The examples show that the model is able to accurately capture the cracking and healing behaviour of this type of self-healing material system with good accuracy.
\end{abstract}

Keywords: Finite element method, Self-healing, Embedded strong discontinuity, Quasi-brittle materials

\section{Introduction}

The formation of cracks in quasi-brittle materials such as concrete produces a degradation in mechanical performance in terms of both stiffness and strength. In addition to this, the presence of cracks leads to significant durability problems, such as reinforcement corrosion and calcium leaching [1]. Self-healing systems are designed to mitigate these issues by introducing crack 'healing' mechanisms into the material that result in a recovery of both mechanical performance and durability properties.

There is now a significant body of work on the numerical simulation of self-healing systems [2-19], as highlighted in a recent review article [20]. The numerical treatment of damage-healing behaviour in mechanical self-healing models has varied, with many utilising a continuum damage-healing mechanics framework (e.g. [5, 7]). Alternative approaches have included a model based on micromechanical theories [11], the discrete element method (DEM) [13], the extended finite element method (XFEM) [12] and embedded discontinuity elements (EFEM) [17]. In addition to this, the treatment of the healing itself has varied, ranging from treating the healing as a thermodynamic

(c) The Author(s) 2020. This article is licensed under a Creative Commons Attribution 4.0 International License, which permits use, sharing adaptation, distribution and reproduction in any medium or format, as long as you give appropriate credit to the original author(s) and the source, provide a link to the Creative Commons licence, and indicate if changes were made. The images or other third party material in this article are included in the article's Creative Commons licence, unless indicated otherwise in a credit line to the material. If material is not included in the article's Creative Commons licence and your intended use is not permitted by statutory regulation or exceeds the permitted use, you will need to obtain permission directly from the copyright holder. To view a copy of this licence, visit http://creativeco mmons.org/licenses/by/4.0/. 
potential, with an associated healing surface [5], to modelling the healing as a chemical reaction such as curing of the healing agent [7] or further hydration in the autogenous healing of cementitious materials [6].

The treatment of cracks in the above methods may be categorised as, (i) discrete crack methods that simulate the separation between elements directly, (ii) strong-discontinuity approaches that maintain the background mesh but add enhanced fields to represent cracks and, (iii) smeared crack approaches that 'smear' the crack displacement jumps over elements [21-24]. Smeared approaches are convenient to implement and can represent bands of diffuse cracks that are found in materials such as concrete prior to the formation of dominant cracks but cannot directly simulate localised failure and can suffer from stress-locking. Discrete approaches provide accurate descriptions of cracks but are not computationally convenient, particularly when multiple cracks occur, as in reinforced concrete members. Strong-discontinuity methods provide a good compromise between the two approaches because they are -in general- computationally convenient yet still provide an explicit representation of cracks; including the location, geometry and crack opening [22-28]. This is particularly important for modelling self-healing systems, as this information is required for the simulation of the transport of healing agents in discrete cracks.

Belytschko and Black [25] (see also Moës et al. [26]) developed the strong-discontinuity-based extended finite element method (XFEM), in which cracks are allowed to cross the mesh arbitrarily and the discontinuities in the displacement field are captured using enrichment functions and additional nodal degrees of freedom. The method makes use of a local partition of unity $[25,29,30]$ and enrichment functions that are usually chosen a priori based on the physics of the problem. In the case of cracks, a step function has been employed for elements completely crossed by a crack [26], whilst additional enrichments have been included at the crack tip [31]. Since this early work on XFEM, the method has been employed for a range of problems involving both strong and weak discontinuities [32, 33]. The generalized finite element method (GFEM), introduced by Strouboulis et al. [34-36] and Duarte et al. [27], at a similar time, is also a partition of unity method [29,37-39] that introduces additional functions (or enrichments) to improve the accuracy of the solution. The enrichment of the finite element space allows discontinuities to be captured internal to the element. In the early work on GFEM, the enrichments of the approximation space were global rather than local [40], although GFEM models incorporating local enrichments appeared soon after [27]. More recently, the names GFEM and XFEM have been used interchangeably [40].

Hansbo and Hansbo [41, 42] proposed an unfitted finite element method, known as CutFEM [43] or the phantom node method [28], for capturing discontinuities internal to the element. The method consists of replacing every element, $K$, which is crossed by a discontinuity that divides the element into two parts, $\Omega_{1}$ and $\Omega_{2}$, with two overlapping elements, $K_{1}$ and $K_{2}$. The new elements are each assigned to a part of the divided element and introduce additional nodes (termed phantom or ghost nodes [28, 44]), allowing the use of the standard basis functions. This leads to a continuous solution in each overlapping element, $u_{1}$ and $u_{2}$, whilst the supposition of these elements allows for a discontinuous solution in the domain of interest (defined for the element $K$ as $u=u_{1} \forall \boldsymbol{x} \in \Omega_{1} \wedge u=u_{2} \forall \boldsymbol{x} \in \Omega_{2}$ ). For an overview of the method, the interested reader 
is referred to Burman et al. [43]. Like XFEM, models utilising CutFEM have been developed for a variety of problems (see for example [45-48]).

An alternative approach to the nodal based enrichment of XFEM, GFEM and CutFEM is to use an element-based enrichment, EFEM. In EFEM, the additional degrees of freedom can be eliminated at the element level using static condensation [49], which leads to a global system of equations comprising only the original degrees of freedom. A comparative study on the two approaches was presented by Oliver et al. [50], who found that EFEM was computationally more efficient than XFEM. Ortiz et al. [51] presented the first work incorporating a weak discontinuity to capture shear bands, whilst Dvorkin et al. [52] presented the first model incorporating a strong discontinuity to capture strain localisation. There are now a range of EFEM models that can be found in the literature $[17,49,51-67]$, with significant developments having been made. A full review of the first decade of research on EFEM is provided in Jirasek [68].

A formulation that allowed for non-constant displacement jumps in each element and a global or local approach, depending on whether or not static condensation was employed, was presented in Alfaiate et al. [49]. The former ensures continuity of the displacement jumps, at the cost of increasing the size of the global system of equations, whilst the latter requires the jumps to be averaged at coincident crack nodes. In Dias-daCosta et al. [58-60] the generalised strong discontinuity approach GSDA was presented. The model employed a global approach and allowed for both non-constant displacement jumps and stretching. Linder and Armero [61,62] developed a new embedded discontinuity element that allowed for branching cracks in a T-shape pattern. The new element was employed in the simulation of a dynamic fracture benchmark problem and showed good agreement with experimental and numerical results. Dynamic effects were also considered in the work of Saksala et al. [64, 65], who developed a 3D model combining continuum visco-damage for the pre-peak response with the embedded discontinuity model for the post-peak response. The model is rate-dependent and employs an explicit time integration to boost computational efficiency. Lu et al. [67] presented a model combining a multi-scale finite element method (MsFEM) [69] with the EFEM for discontinuities in saturated porous media (see also Lu et al. [66] for modelling cracks in solids). The model combines the ability of EFEM to capture localisations with the computational efficiency of MsFEM. Numerical examples were presented which showed that the proposed method gave accurate predictions when compared with EFEM, with a greater computational efficiency. A model for self-healing quasi-brittle materials was presented by Zhang and Zhuang [17]. The authors utilised an EFEM approach that allowed for crack opening and crack sliding. The model used a reduced integration for cracked elements and smeared the displacement jump across the crack band. The healing was introduced at the constitutive level into the traction-separation law, with an Arrhenius-type law being employed for the solidification of the healing agent.

In this study, the EFEM approach is employed and a specialised element is presented that takes healing into account at the element level. The element-based strong-discontinuity approach for simulating both cracking and healing is preferred to the smeared approach of Zhang and Zhuang [17] because the former provides a more accurate description of the crack geometry and crack displacements, which is particularly important in the present work, in which the mechanical model is coupled to a transport model 
for the flow of healing agents through the discrete cracks [19]. The approach adopted for this work ensures that the healing is consistent with the internal degrees of freedom introduced to represent the crack and allows for a variational derivation. A cohesive zone approach with a damage-healing constitutive model is employed, whilst equilibrium over the crack plane is satisfied in a weak sense using a local variation in the internal degrees of freedom. The element allows for different healing mechanisms and for overlapping cycles of damage and healing when combined with a suitable crack plane model, such as that employed in the present work [70].

The principal contributions of this work are, (i) a new element with healing degrees of freedom linked to a unique damage-healing constitutive model, (ii) the implementation of the element within a new coupled transport-mechanical computational framework, and (iii) validations using new experimental data and idealisations.

The layout of the remainder of this paper is as follows:

- "Theoretical basis for the element" section describes the theoretical formulation of the element including the introduction of healing:

- "Healing agent transport and curing model" section describes the healing agent transport and curing model employed in this study:

- "Crack-plane constitutive model" section describes the crack-plane constitutive model employed in this study:

- "Example problems" section presents a convergence test and two example problems concerning a self-healing concrete with a vascular healing system filled with cyanoacrylate, the first being based on a direct tension test and the second an L-shaped specimen:

- "Conclusions" section gives some conclusions.

\section{Theoretical basis for the element}

\section{A quadrilateral finite element with an embedded strong discontinuity}

The aim of this section is to present the formulation of a quadrilateral finite element (Q4) capable of describing embedded crack phenomena governed by any thermodynamically valid inelastic constitutive relationship. The starting point will be the general Q4 bilinear quadrilateral element to which is added a straight discontinuity, which crosses the element at an angle $\psi$, as illustrated in Fig. 1.

The problem domain is defined as $\Omega \in \mathbb{R}^{2}$ with boundary $\Gamma$. The total virtual work $(\delta \Pi)$ for this domain may be written as follows, noting that $\delta$ denotes a virtual quantity:

$$
\begin{array}{cr}
\delta \Pi=\int_{\Omega} \delta \boldsymbol{\varepsilon}: \boldsymbol{\sigma} \mathrm{d} \Omega-\int_{\Omega} \delta \mathbf{u} \cdot \mathbf{F}_{B} \mathrm{~d} \Omega-\int_{\partial \Omega} \delta \mathbf{u} \cdot \mathbf{F}_{\partial \Omega} \mathrm{d} \Omega=0, & \text { in } \Omega, \\
\mathbf{u}=\mathbf{u}_{D} & \text { on } \Gamma_{D}, \\
\overrightarrow{\mathbf{n}} \cdot \boldsymbol{\sigma}=\mathbf{g}_{D} & \text { on } \Gamma_{N} .
\end{array}
$$

where $\mathbf{x}$ is the Cartesian basis, $\mathbf{u}$ denotes the displacement vector; $t \in\left[t_{0}, T\right]$ is the time., $\Gamma_{D}$ and $\Gamma_{\mathrm{N}}$ are the complementary subsets of the boundary where the prescribed displacements $\mathbf{u}_{D}(\mathbf{x}, t)$ and tractions $\mathbf{g}_{D}(\mathbf{x}, t)$ are applied, $\boldsymbol{\varepsilon}(\mathbf{u})$ is the strain tensor, $\boldsymbol{\sigma}(\boldsymbol{\varepsilon})$ is the stress tensor, $\mathbf{F}_{B}(\mathbf{x}, t)$ and $\mathbf{F}_{\partial \Omega}(\mathbf{x}, t)$ describe the external body and surface forces respectively, and $\overrightarrow{\mathbf{n}}$ is a unit vector on the boundary. 


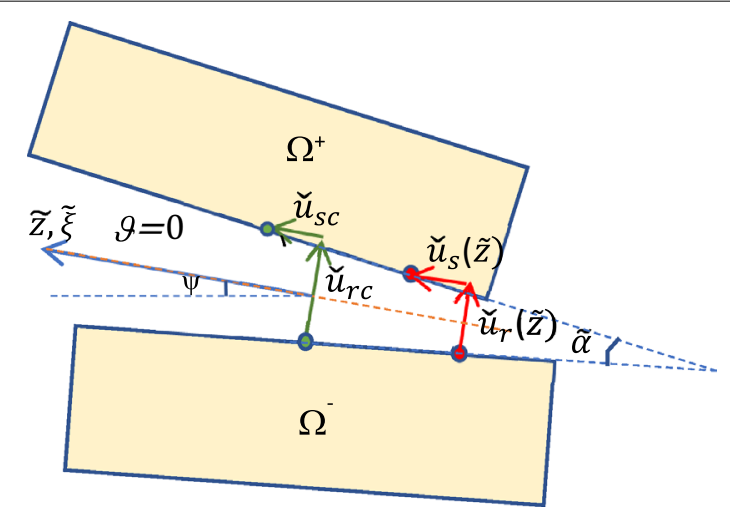

Fig. 1 Inelastic relative displacement of $\Omega_{k}^{+}$with respect to $\Omega_{k}^{-}$in the strong discontinuity element

If the domain $\Omega$ is divided into standard Q4 elements, it is assumed that within each elemental subdomain $\left(\Omega_{e}\right)$ any displacement can be linearly interpolated from the values at the nodes (vector $\overline{\mathbf{u}}$ ) through the shape functions (i.e. $\mathbf{u}(\mathbf{x})=\mathbf{N}(\mathbf{x}) \mathbf{u}_{e}$ ). In this paper, direct tensor notation is used for Eq. (1) and the continuum constitutive equations but matrix notation is employed when the discretised form of equations and crack-plane constitutive relationships are considered.

A method for introducing a discontinuity into the element is now described.

Let $\vartheta\left(\mathbf{x} ; \mathbf{x}_{C}, \psi\right)$ be the signed-distance equation describing the linear discontinuity (see Fig. 1), where $\mathbf{x}_{C}$ stands for the central point of the crack within the element. Two subdomains $\Omega_{k}^{+}$and $\Omega_{k}^{-}$can be defined as $\mathbf{x} \in \Omega_{e}^{+} \forall \vartheta(\mathbf{x}) \geq 0$ and $\mathbf{x} \in \Omega_{e}^{-} \forall \vartheta(\mathbf{x})<0$. Then, using the Heaviside function $H_{\vartheta}$ placed at this discontinuity, the elemental displacements can be expressed as the sum of two functions $\mathbf{u}_{e}$ and $\llbracket \mathbf{u} \rrbracket$, the later representing the jump. In other words:

$$
\mathbf{u}(\mathbf{x}, t)=\mathbf{u}_{e}(\mathbf{x}, t)+H_{\vartheta} \llbracket \mathbf{u} \rrbracket(\mathbf{x}, t)
$$

In the present formulation, the continuum material is assumed to be elastic.

The inelastic displacement associated with the discontinuity is extracted from a narrow band of material of finite width (See Fig. 2). This narrow band material containing the crack will be termed 'the crack-plane', which is defined in the orthonormal (local) basis $\{\overrightarrow{\mathbf{r}}, \overrightarrow{\mathbf{s}}\}$, with $\overrightarrow{\mathbf{r}}$ being normal to the crack-plane. The local crack-plane relative-displacement vector $(\tilde{\mathbf{u}})$ is the sum of an inelastic part $(\tilde{\mathbf{u}})$, which contains the crack opening and sliding displacements, and an elastic part $\left(\tilde{\mathbf{u}}^{e}\right)$. The total crackplane relative displacements, including those of the elastic band of material either side of the discontinuity, are illustrated in Fig. 2.

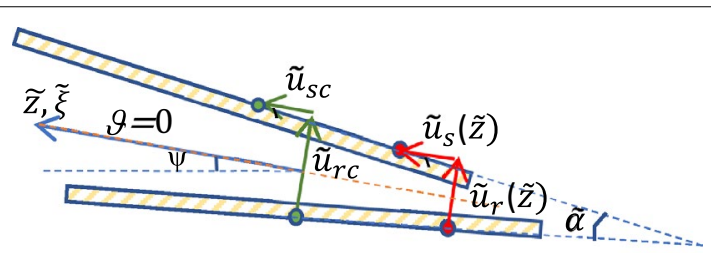

Fig. 2 Crack-plane relative-displacements including elastic-band of material 
The stress in the continuum part of the element $\left(\Omega_{k}^{+} \cup \Omega_{k}^{-}\right)$is required, and is given by:

$$
\sigma=\mathbf{D}:\left(\varepsilon-\varepsilon_{\vartheta}\right)
$$

where $\mathbf{D}$ is the elasticity tensor and $\boldsymbol{\varepsilon}_{\vartheta}\left(\widetilde{\mathbf{w}}, \mathbf{x}_{j}\right)$ is the strain in the continuum part of the element caused by the displacement jump at the discontinuity, noting that $\widetilde{\mathbf{w}}$ is defined below.

The second term requires the tractions and displacements across the discontinuity. In the present approach, the latter are derived from the following crack-plane vector, which contains crack opening and sliding displacement components, as well as the rotation at the mid-point of the crack within an element (i.e. at $\mathbf{x}=\mathbf{x}_{C}$ );

$$
\breve{\mathbf{w}}=\left[\breve{u}_{r, C}, \breve{u}_{s, C}, \breve{\alpha}\right]^{T}
$$

The opening and sliding displacement components at a position $\tilde{Z}$ along the crack (see Fig. 1) are obtained from $\mathbf{w}$ as follows;

$$
\breve{\mathbf{u}}=\tilde{\Lambda} \breve{\mathbf{w}}
$$

in which $\tilde{\Lambda}(\tilde{Z})=\left[\begin{array}{lll}1 & 0 & \tilde{Z} \\ 0 & 1 & 0\end{array}\right]$ and, in the present $2 \mathrm{D}$ case, $\breve{\mathbf{u}}^{\mathrm{T}}=\left[\begin{array}{cc}\breve{u}_{r} & \breve{u}_{s}\end{array}\right]$.

Where, the variable $\tilde{Z} \in 0.5 \cdot\left[-\tilde{l}_{\vartheta}, \tilde{l}_{\vartheta}\right]$ spans the crack length $\tilde{l}_{\vartheta}$ in the same direction as $\overrightarrow{\mathbf{s}}$; and $t_{h k}$ is the out-of-plane thickness of the crack band.

The work conjugate vector to $\widetilde{\mathbf{w}}$, which contains force and moment terms, is obtained by integrating the tractions along the length of the discontinuity, as follows:

$$
\tilde{\mathbf{F}}=t_{h k}\left[\begin{array}{c}
\int_{-C K}^{l_{C K} / 2} \tilde{\sigma}_{r} d \tilde{Z} \\
\int_{-l_{C K} / 2}^{l_{C K} / 2} \tilde{\sigma}_{s} d \tilde{Z} \\
\int_{-l_{C K} / 2}^{l_{C K} / 2} \tilde{\sigma}_{r} \tilde{Z} d \tilde{Z}
\end{array}\right]
$$

The tractions applied to the crack plane of material are equal to those applied across the discontinuity, thus $\tilde{\sigma}=\widetilde{\sigma}$ and $\tilde{\mathbf{F}}=\breve{\mathbf{F}}$.

In order to derive $\boldsymbol{\varepsilon}_{\vartheta}$, an expression is needed for the displacement in the positive part of the elements (i.e. $\mathbf{x} \in \Omega^{+}$) due solely to the relative-displacement across the discontinuity (i.e. $\mathbf{u}_{\vartheta}(\mathbf{x})$ ). This is given by the following expression, which is based on the assumption that the rotation $(\tilde{\alpha})$ is small (See Fig. 1):

$$
\mathbf{u}_{\vartheta}(\mathbf{x})=\left[\begin{array}{ccc}
-\sin (\psi) & -\cos (\psi) & R_{x}\left(\mathbf{x} ; \tilde{\alpha}, \mathbf{x}_{C}, H_{\vartheta}\right) \\
\cos (\psi) & -\sin (\psi) & R_{y}\left(\mathbf{x} ; \tilde{\alpha}, \mathbf{x}_{C}, H_{\vartheta}\right)
\end{array}\right] \cdot \breve{\mathbf{w}}=\mathbf{T}_{\mathbf{w}}\left(\mathbf{x} ; \psi, \tilde{\alpha}, \mathbf{x}_{C}, H_{\vartheta}\right) \cdot \breve{\mathbf{w}}
$$

in which

$$
\mathbf{R}\left(\mathbf{x}_{j} ; \tilde{\alpha}, \mathbf{x}_{C}, H_{\vartheta}\right)=H_{\vartheta}(\mathbf{x}) \cdot \overrightarrow{\mathbf{t}} \times\left(\mathbf{x}_{j}-\mathbf{x}_{C}\right)
$$

The operator $\mathbf{T}_{\mathbf{w}}\left(\mathbf{x} ; \psi, \tilde{\alpha}, \mathbf{x}_{C}, H_{\vartheta}\right)$ maps the rigid body motion induced by the discontinuity to the parent element nodes and the introduction of $H_{\vartheta}$ ensures that vector $\mathbf{u}_{\vartheta}(\mathbf{x})$ is only non-zero in the positive part of the element. By considering only the rigid body motions, it is implicitly assumed the enhanced strain in the continuum is null. 
This means that integrations over multiple element zones are avoided [59], which makes implementation more straightforward. However, this comes at the expense of restricting the shear jumps to being constant along the discontinuity in each element [59]. In some shear dominated problems, this restriction may mean that a finer mesh would be required to achieve the same level of accuracy compared to a solution using elements with a tangential relative stretching mode [63], but this issue is not believed to be significant for the problems considered in this paper.

$\boldsymbol{\varepsilon}_{\vartheta}$ is then obtained by applying Eq. (7) to the element nodal displacements $\mathbf{u}_{\vartheta}\left(\mathbf{x}_{j}\right)(j$ denoting the element local node number) and by employing the standard strain-displacement matrix $\mathbf{B}(\mathbf{x})$ derived from the element shape functions $\mathcal{N}(\mathbf{x})$. This gives the following equation;

$$
\boldsymbol{\varepsilon}_{\vartheta}=\mathbf{M} \breve{\mathbf{w}}
$$

in which $\mathbf{M}(\mathbf{x})=\mathbf{B}(\mathbf{x}) \mathbf{T}_{\mathbf{w}}\left(\mathbf{x}_{j}\right)$.

The discontinuity is assumed to follow the basic elastic-damage cohesive crack constitutive relationship given in Jefferson et al. [71]. This is based on damage mechanics and gives the following relationship between the crack-plane tractions and relative displacements;

$$
\tilde{\sigma}=(1-\tilde{\omega}) \tilde{\mathbf{k}}^{e} \tilde{\mathbf{u}}=\tilde{\mathbf{k}} \tilde{\mathbf{u}}
$$

in which $\tilde{\sigma}=\left[\begin{array}{ll}\tilde{\sigma}_{r} & \tilde{\sigma}_{s}\end{array}\right]^{\mathrm{T}} ; \tilde{\mathbf{u}}=\left[\begin{array}{ll}\tilde{u}_{r} & \tilde{u}_{s}\end{array}\right]^{\mathrm{T}} ; \tilde{\mathbf{k}}=(1-\tilde{\omega}(\tilde{\zeta})) \tilde{\mathbf{k}}^{e} ; \tilde{\mathbf{k}}^{e}=\left[\begin{array}{cc}\tilde{k}_{r} & 0 \\ 0 & \tilde{k}_{s}\end{array}\right] ; \tilde{k}_{r}$ and $\tilde{k}_{s}$ the normal and shear elastic moduli respectively for the crack-plane; $\tilde{\omega} \in[0,1]$ is the crackplane damage variable, which depends on the damage evolution parameter $(\tilde{\zeta})$, according to the functions given in Sect. 4. It is also noted that subscript $e$ denotes an element quantity and superscript $e$ refers to an elastic entity.

Using Eq. (9) in Eq. (6) leads to the following relationship between the crack-plane displacement and force vectors:

$$
\tilde{\mathbf{F}}=\tilde{\mathbf{K}} \tilde{\mathbf{w}}
$$

in which $\mathbf{K}=t_{h k} \cdot\left[\begin{array}{c:c:c}\int_{-1}^{1}(1-\tilde{\omega}) \tilde{k_{r}} \tilde{J} d \tilde{\xi} & 0 & \int_{-1}^{1}(1-\tilde{\omega}) \tilde{k_{r}} \tilde{Z} \tilde{J} d \tilde{\xi} \\ \hdashline 0 & \int_{-1}^{1}(1-\tilde{\omega}) \tilde{k}_{s} \tilde{J} d \tilde{\xi} & 0 \\ \hdashline \int_{-1}^{1}(1-\tilde{\omega}) \tilde{k_{r}} \tilde{Z} \tilde{J} d \tilde{\xi} & 0 & \int_{-1}^{1}(1-\tilde{\omega}) \tilde{k}_{r} \tilde{Z}^{2} \tilde{J} d \tilde{\xi}\end{array}\right] \tilde{\xi} \in[-1,1]$ is the parametric coordinate along the discontinuity and $\tilde{J}=d \tilde{Z} / d \tilde{\xi}$ the resultant Jacobian.

The relationship between the crack-plane and inelastic displacement vectors is given by;

$$
\breve{\mathbf{w}}=\tilde{\mathbf{w}}-\tilde{\mathbf{K}} e^{-1} \tilde{\mathbf{F}}=\tilde{\mathbf{w}}-\tilde{\mathbf{K}} e^{-1} \tilde{\mathbf{K}} \tilde{\mathbf{w}}==\left(\mathbf{I}_{3}-\tilde{\mathbf{K}}^{e^{-1}} \tilde{\mathbf{K}}\right) \tilde{\mathbf{w}}=\breve{\mathbf{I}} \tilde{\mathbf{w}}
$$

in which $\mathbf{I}_{3}$ is the rank 3 identity matrix. 
Making use of Eqs. (1), (3) and (11), the total virtual work may be written as the sum of the virtual work of the continuum part of the element and that of the discontinuity, as follows:

$$
\delta \Pi_{e}=\int_{\Omega_{e l}}\left(\delta \boldsymbol{\varepsilon}-\delta \boldsymbol{\varepsilon}_{\vartheta}\right)^{\mathrm{T}} \mathbf{D}\left(\boldsymbol{\varepsilon}-\boldsymbol{\varepsilon}_{\vartheta}\right) \mathrm{d} \Omega_{e}+\delta \stackrel{\mathbf{w}}{\mathrm{T}}^{\mathbf{F}}-\delta \mathbf{u}_{e}^{\mathrm{T}} \mathbf{F}_{e}=0
$$

where $\mathbf{F}_{e}=\int_{\Omega_{e}} \delta \mathbf{u}_{e}^{\mathrm{T}} \mathbf{F}_{B_{e l}} \mathrm{~d} \Omega+\int_{\partial \Omega_{e}} \delta \mathbf{u}_{e}^{\mathrm{T}} \mathbf{F}_{\partial \Omega_{e}} \mathrm{~d} \Omega_{e}$.

Using (9) in (13) results in the following revised virtual work equation;

$$
\delta \Pi_{e}=\int_{\Omega_{e l}}\left(\delta \mathbf{u}_{e}^{\mathrm{T}} \mathbf{B}^{\mathrm{T}}-\delta \breve{\mathbf{w}}^{\mathrm{T}} \mathbf{M}^{\mathrm{T}}\right) \mathbf{D}\left(\mathbf{B} \mathbf{u}_{e}-\mathbf{M} \breve{\mathbf{w}}\right) \mathrm{d} \Omega_{e}+\delta \breve{\mathbf{w}}^{\mathrm{T}} \tilde{\mathbf{F}}-\delta \mathbf{u}_{e}^{\mathrm{T}} \mathbf{F}_{e}=0
$$

Since the constitutive relationships (10) and (11) are written in terms of the total crack-plane displacement vector, it proves convenient to express (14) in terms of $\tilde{\mathbf{w}}$ rather then $\widetilde{\mathbf{w}}$. This is accomplished by using Eqs. (9) and (12), as follows;

$$
\boldsymbol{\varepsilon}_{\vartheta}=\tilde{\mathbf{M}} \tilde{\mathbf{w}}
$$

in which $\tilde{\mathbf{M}}(\mathbf{x})=\mathbf{B}(\mathbf{x}) \mathbf{T}_{\mathbf{w}}\left(\mathbf{x}_{j}\right) \breve{\mathbf{I}}$.

Using Eq. (15) and employing Eq. (11) gives;

$$
\delta \Pi_{e}=\int_{\Omega_{e l}}\left(\delta \mathbf{u}_{e}^{\mathrm{T}} \mathbf{B}^{\mathrm{T}}-\delta \tilde{\mathbf{w}}^{\mathrm{T}} \tilde{\mathbf{M}}^{\mathrm{T}}\right) \mathbf{D}\left(\mathbf{B} \mathbf{u}_{e}-\tilde{\mathbf{M}} \tilde{\mathbf{w}}\right) \mathrm{d} \Omega_{e}+\delta \tilde{\mathbf{w}}^{\mathrm{T}} \overline{\mathbf{K}} \tilde{\mathbf{w}}-\delta \mathbf{u}_{e}^{\mathrm{T}} \mathbf{F}_{e}=0
$$

where $\overline{\mathbf{K}}=\breve{\mathbf{I}}^{\mathrm{T}} \tilde{\mathbf{K}}$.

Equilibrium between the tractions across the discontinuity and the stresses in the adjacent continuum is considered in a weak sense by assuming that the element nodal displacements are fixed and that there is a virtual change in $\tilde{\mathbf{w}}$, which leads to;

$$
\delta \Pi_{e w}=\int_{\Omega_{e}}\left(-\delta \tilde{\mathbf{w}}^{\mathrm{T}} \tilde{\mathbf{M}}^{\mathrm{T}}\right) \mathbf{D}\left(\mathbf{B} \mathbf{u}_{e}-\tilde{\mathbf{M}} \tilde{\mathbf{w}}\right) \mathrm{d} \Omega_{e}+\delta \tilde{\mathbf{w}}^{\mathrm{T}} \overline{\mathbf{K}} \tilde{\mathbf{w}}=0
$$

from which the following relationship between the crack-plane displacement vector and element nodal displacements can be derived;

$$
\tilde{\mathbf{w}}=\mathbf{C} \mathbf{u}_{e}
$$

in which $\mathbf{C}=\left[\int_{\Omega_{e}} \tilde{\mathbf{M}}^{\mathrm{T}} \mathbf{D} \tilde{\mathbf{M}} \mathrm{d} \Omega_{e}+\overline{\mathbf{K}}\right]^{-1} \int_{\Omega_{e}} \tilde{\mathbf{M}}^{\mathrm{T}} \mathbf{D B} \mathrm{d} \Omega_{e}$

Using Eq. (18) in (16) gives;

$$
\delta \Pi_{e}=\delta \mathbf{u}_{e}^{\mathrm{T}} \int_{\Omega_{e l}}\left(\mathbf{B}^{\mathrm{T}}-\mathbf{C}^{\mathrm{T}} \tilde{\mathbf{M}}^{\mathrm{T}}\right) \mathbf{D}(\mathbf{B}-\tilde{\mathbf{M}} \mathbf{C}) \mathrm{d} \Omega_{e} \mathbf{u}_{e}+\delta \mathbf{u}_{e}^{\mathrm{T}} \mathbf{C}^{\mathrm{T}} \overline{\mathbf{K}} \mathbf{C} \mathbf{u}_{e}-\delta \mathbf{u}_{e}^{\mathrm{T}} \mathbf{F}_{e}=0 .
$$

The element stiffness relationship may then be obtained by cancelling the common virtual displacements and rearranging Eq. (19), as follows: 


$$
\mathbf{F}_{e}=\mathbf{K}_{S D e} \mathbf{u}_{e}
$$

where $\mathbf{K}_{S D e}=\int_{\Omega_{e l}}\left(\mathbf{B}^{\mathrm{T}}-\mathbf{C}^{\mathrm{T}} \tilde{\mathbf{M}}^{\mathrm{T}}\right) \mathbf{D}(\mathbf{B}-\tilde{\mathbf{M}} \mathbf{C}) \mathrm{d} \Omega_{e}+\mathbf{C}^{\mathrm{T}} \overline{\mathbf{K}} \mathbf{C}$.

In this approach, equilibrium across the discontinuity and the crack-plane displacements are defined at the element level (see Eq. (18)). Whilst this means that there is no continuity of the crack-plane displacements across element boundaries (though the mean values at coincident nodes could be adopted in the constitutive law [49]), it ensures that the bandwidth of the global stiffness matrix remains constant [58]. We also note that the crack path used in the discrete flow computations is smoothed by averaging the crack openings from adjacent elements at common boundaries.

\section{Extension to damage-healing}

To account for healing, the local constitutive relationship (10) is modified by adding a term to account for the damaged material that has been restored. The proportion of material that is healed at time $t$ is given by $\tilde{h}(t) \in[0, \tilde{\omega}]$ and the healing displacement $\left(\tilde{\mathbf{u}}_{h}\right)$ is introduced to ensure that an increment of healing takes place under constant stress, which ensures that a healing step doesn't violate the second law of thermodynamics (See Jefferson et al. [20]). Various forms of healing model are described in Jefferson et al. [20] and a specific model (see "Healing agent transport and curing model" and "Crack-plane constitutive model" sections for an overview) is presented in Freeman and Jefferson [19] and Jefferson et al. [70]. The aim here is to show how a general form of local healing model may be incorporated into the element with an embedded strong discontinuity.

$$
\tilde{\sigma}=(1-\tilde{\omega}) \tilde{\mathbf{k}}^{e} \tilde{\mathbf{u}}+\tilde{h} \tilde{\mathbf{k}}^{h e}\left(\tilde{\mathbf{u}}-\tilde{\mathbf{u}}_{h}\right)
$$

The elastic-damage-healing counterpart to Eq. (11) may be obtained from Eq. (19), as follows:

$$
\tilde{\boldsymbol{F}}=\tilde{\boldsymbol{K}} \tilde{\boldsymbol{w}}+\tilde{\boldsymbol{K}}_{h}\left(\tilde{\boldsymbol{w}}-\tilde{\boldsymbol{w}}_{h}\right)
$$

in which

$$
\tilde{\mathbf{K}}_{h}=t_{h k} \cdot\left[\begin{array}{c:c:c}
\int_{-1}^{1} \tilde{h} \cdot k_{r}^{h} \tilde{J} d \tilde{\xi} & 0 & \int_{-1}^{1} \tilde{h} \cdot k_{r}^{h} \tilde{Z} \tilde{J} d \tilde{\xi} \\
\hdashline 0 & \int_{-1}^{1} \tilde{h} \cdot k_{s}^{h} \tilde{J} d \tilde{\xi} & 0 \\
\hdashline \int_{-1}^{1} \tilde{h} \cdot k_{r}^{h} \tilde{Z} \tilde{J} d \tilde{\xi} & 0 & \int_{-1}^{1} \tilde{h} \cdot k_{r}^{h} \tilde{Z}^{2} \tilde{J} d \tilde{\xi}
\end{array}\right]
$$

The inelastic component of displacement is now given by:

$$
\breve{\mathbf{w}}=\left(\mathbf{I}_{3}-\tilde{\mathbf{K}}^{e^{-1}}\left(\tilde{\mathbf{K}}+\tilde{\mathbf{K}}_{h}\right)\right) \tilde{\mathbf{w}}+\tilde{\mathbf{K}}^{e^{-1}} \tilde{\mathbf{K}}_{h} \tilde{\mathbf{w}}_{h}
$$

and the strain change due to the relative displacements across the discontinuity is;

$$
\boldsymbol{\varepsilon}_{\vartheta}=\mathbf{M}_{w} \tilde{\mathbf{w}}+\mathbf{M}_{w} \tilde{\mathbf{w}}_{h}
$$


in which $\mathbf{M}_{w}=\mathbf{M}\left(\mathbf{I}_{3}-\tilde{\mathbf{K}}^{e^{-1}}\left(\tilde{\mathbf{K}}+\tilde{\mathbf{K}}_{h}\right)\right)$ and $\mathbf{M}_{h}=\tilde{\mathbf{K}}^{e^{-1}} \tilde{\mathbf{K}}_{h}$.

Since an increment of healing takes place under a zero change in stress, it may be assumed that $; \delta \breve{\boldsymbol{w}}=\left(\mathbf{I}_{3}-\tilde{\mathbf{K}}^{-1}\left(\tilde{\boldsymbol{K}}+\tilde{\boldsymbol{K}}_{h}\right)\right) \delta \tilde{\boldsymbol{w}}$ and $\delta \boldsymbol{\varepsilon}_{\vartheta}=\mathbf{M}_{w} \delta \tilde{\mathbf{w}}$

The virtual work equation for the damage-healing is obtained by using Eqs. (22-24) in Eq. (14), as follows;

$$
\begin{aligned}
\delta \Pi_{e}= & \int_{\Omega_{e l}}\left(\delta \mathbf{u}_{e}^{\mathrm{T}} \mathbf{B}^{\mathrm{T}}-\delta \tilde{\mathbf{w}}^{\mathrm{T}} \mathbf{M}_{w}^{\mathrm{T}}\right) \mathbf{D}\left(\mathbf{B} \mathbf{u}_{e}-\mathbf{M}_{w} \tilde{\mathbf{w}}-\mathbf{M}_{h} \tilde{\mathbf{w}}_{h}\right) \mathrm{d} \Omega_{e} \\
& +\delta \tilde{\mathbf{w}}^{\mathrm{T}}\left(\tilde{\mathbf{K}}_{w w} \tilde{\mathbf{w}}-\tilde{\mathbf{K}}_{w h} \tilde{\mathbf{w}}_{h}\right)-\delta \mathbf{u}_{e}^{\mathrm{T}} \mathbf{F}_{e}=0
\end{aligned}
$$

in which $\tilde{\mathbf{K}}_{w w}=\left(\mathbf{I}_{3}-\tilde{\mathbf{K}}^{e^{-1}}\left(\tilde{\mathbf{K}}+\tilde{\mathbf{K}}_{h}\right)\right)^{\mathrm{T}}\left(\tilde{\mathbf{K}}+\tilde{\mathbf{K}}_{h}\right)$ and $\tilde{\mathbf{K}}_{w h}=\left(\mathbf{I}_{3}-\tilde{\mathbf{K}}^{e^{-1}}\left(\tilde{\mathbf{K}}+\tilde{\mathbf{K}}_{h}\right)\right)^{\mathrm{T}} \tilde{\mathbf{K}}_{h}$

The equivalence of Eq. (17), which is used to satisfy equilibrium between the crack and the continuum for the damage-healing case, is

$$
\delta \Pi_{e}=\int_{\Omega_{e l}}\left(-\delta \tilde{\mathbf{w}}^{\mathrm{T}} \mathbf{M}_{w}^{\mathrm{T}}\right) \mathbf{D}\left(\mathbf{B} \mathbf{u}_{e}-\mathbf{M}_{w} \tilde{\mathbf{w}}-\mathbf{M}_{h} \tilde{\mathbf{w}}_{h}\right) \mathrm{d} \Omega_{e}+\delta \tilde{\mathbf{w}}^{\mathrm{T}}\left(\tilde{\mathbf{K}}_{w w} \tilde{\mathbf{w}}-\tilde{\mathbf{K}}_{w h} \tilde{\mathbf{w}}_{h}\right)=0
$$

From which the following relationship may be derived;

$$
\tilde{\mathbf{w}}=\mathbf{C}_{u} \mathbf{u}_{e}+\mathbf{C}_{h} \tilde{\mathbf{w}}_{h}
$$

in which $\mathbf{C}_{u}=\left[\int_{\Omega_{e l}} \mathbf{M}_{w}^{\mathrm{T}} \mathbf{D} \mathbf{M}_{w} \mathrm{~d} \Omega_{e}+\tilde{\mathbf{K}}_{w w}\right]^{-1} \int_{\Omega_{e l}} \mathbf{M}_{w}^{\mathrm{T}} \mathbf{D B} \mathrm{d} \Omega_{e}$ and $\mathbf{C}_{h}=\int_{\Omega_{e l}}-\mathbf{M}_{w}^{\mathrm{T}} \mathbf{D} \mathbf{M}_{h} \mathrm{~d} \Omega_{e}+\tilde{\mathbf{K}}_{w h}$.

Using Eq. (27) in Eq. (25) gives;

$$
\begin{array}{r}
\delta \Pi_{e}=\int_{\Omega_{e l}}\left(\delta \mathbf{u}_{e}^{\mathrm{T}} \mathbf{B}^{\mathrm{T}}-\delta \mathbf{u}_{e}^{\mathrm{T}} \mathbf{C}_{u}^{\mathrm{T}} \mathbf{M}_{w}^{\mathrm{T}}\right) \mathbf{D}\left(\mathbf{B} \mathbf{u}_{e}-\mathbf{M}_{w}\left(\mathbf{C}_{u} \mathbf{u}_{e}+\mathbf{C}_{h} \tilde{\mathbf{w}}_{h}\right)-\mathbf{M}_{h} \tilde{\mathbf{w}}_{h}\right) \mathrm{d} \Omega_{e} \\
+\delta \mathbf{u}_{e}^{\mathrm{T}} \mathbf{C}_{u}^{\mathrm{T}}\left(\tilde{\mathbf{K}}_{w w}\left(\mathbf{C}_{u} \mathbf{u}_{e}+\mathbf{C}_{h} \tilde{\mathbf{w}}_{h}\right)-\tilde{\mathbf{K}}_{w h} \tilde{\mathbf{w}}_{h}\right)-\delta \mathbf{u}_{e}^{\mathrm{T}} \mathbf{F}_{e}=0
\end{array}
$$

from which the following element stiffness relationship may be derived;

$$
\mathbf{K}_{S D e h} \mathbf{u}_{e}=\mathbf{F}_{e}+\mathbf{K}_{e h} \tilde{\mathbf{w}}_{h}
$$

in which

$$
\begin{aligned}
& \mathbf{K}_{S D e h}=\int_{\Omega_{e l}}\left(\mathbf{B}^{\mathrm{T}}-\mathbf{C}_{u}^{\mathrm{T}} \mathbf{M}_{w}^{\mathrm{T}}\right) \mathbf{D}\left(\mathbf{B}-\mathbf{M}_{w} \mathbf{C}_{u}\right) \mathrm{d} \Omega_{e}+\mathbf{C}_{u}^{\mathrm{T}} \tilde{\mathbf{K}}_{w w} \mathbf{C}_{u} \\
& \text { and } \mathbf{K}_{e h}=\int_{\Omega_{e l}}\left(\mathbf{B}^{\mathrm{T}}-\mathbf{C}_{u}^{\mathrm{T}} \mathbf{M}_{w}^{\mathrm{T}}\right) \mathbf{D}\left(\mathbf{M}_{w} \mathbf{C}_{h}+\mathbf{M}_{h}\right) \mathrm{d} \Omega_{e}-\left(\mathbf{C}_{u}^{\mathrm{T}} \tilde{\mathbf{K}}_{w w} \mathbf{C}_{h}-\tilde{\mathbf{K}}_{w h}\right)
\end{aligned}
$$

and where $\tilde{\mathbf{w}}_{h}$ is defined at the element level.

In the following sections the specific healing agent transport, curing and crackplane constitutive models that are employed in this study are described. The authors 
emphasise that the element is general and could be readily combined with other forms of healing model.

\section{Healing agent transport and curing model}

The transport of the healing agent through the crack was calculated using the modified Lucas-Washburn equation of Gardner et al. [72-74] (see also Selvarajoo et al. [75]), that reads:

$$
\dot{z}=\frac{P_{c}\left(1-\beta_{s}\right)+P_{a p p}-\rho g z \sin (\phi)}{\frac{2 \beta_{m}}{\tilde{w}_{r, C}}+\eta}
$$

where $z$ denotes the cumulative flow length in the crack, the superior dot denotes a time derivative, $P_{c}$ is the capillary pressure, $P_{a p p}$ is the applied pressure, $\rho$ is the density, $g$ is the acceleration due to gravity, $\phi$ is the inclination of the crack, $\beta_{s}$ and $\beta_{m}$ are factors to allow for stick-slip of the meniscus and frictional dissipation at the meniscus respectively and $\eta$ is the viscous resistance given by:

$$
\eta=\tilde{w}_{r, C}(z) \int_{0}^{z} \frac{1}{\tilde{w}_{r, C}(x)\left(\frac{\tilde{w}_{r, C}(x) \beta_{w}}{2}+\frac{k(x)}{\mu}\right)} d x
$$

where $\beta_{w}$ is a factor to allow for wall slip, $k=\tilde{w}_{r, C}^{2} / 12$ is the permeability and $\mu$ is the dynamic viscosity of the healing agent.

The capillary pressure is given by the Young-Laplace equation:

$$
P_{c}=\frac{2 \gamma \cos \left(\theta_{d}\right)}{\tilde{w}_{r, C}}
$$

where $\gamma$ denotes the surface tension and $\theta_{d}$ is the dynamic contact angle.

The dynamic contact angle is a function of the meniscus velocity and is described using the relationship of Jiang et al. [76]:

$$
\tanh \left(c_{1}\left(\frac{\dot{z} \mu}{\gamma}\right)^{c_{2}}\right)=\frac{\cos \left(\theta_{s}\right)-\cos \left(\theta_{d}\right)}{\cos \left(\theta_{s}\right)+1}
$$

where $c_{1}$ and $c_{2}$ are constants, and $\theta_{s}$ denotes the static contact angle.

Cyanoacrylate cures through a polymerisation reaction in the presence moisture $[75,77]$. The moisture required for the reaction is transported into the glue from the substrate or the surrounding air. This leads to a reaction front which propagates into the glue, which, experimental evidence shows is diffuse in nature [75, 78]. This can be described by [19]:

$$
\varphi_{h}(x, t)=\frac{1}{2}\left(1-\tanh \left(\left(\frac{2}{\sqrt{\pi}}\right)\left(\frac{x-z(t)-z_{c}}{z_{c}+\sqrt{\frac{z(t)}{z_{c 1}}}}\right)\right)\right)
$$

where $x$ denotes the position measured from the crack face, $t$ is time, $z_{c}$ is a wall factor, $z_{c 1}$ is a diffusion coefficient and $z(t)$ denotes the position of the reaction front.

The propagation of the reaction front is given by $[19,75]$ : 


$$
z(t)=z_{c 0}\left(1-e^{\frac{-t}{\tau}}\right)
$$

where $z_{c 0}$ is the critical reaction front depth and $\tau$ is the characteristic time.

The degree of mechanical healing found in Eq. (21) in the absence of re-damage- is given as the degree of cure at the centre of the crack:

$$
\tilde{h}=\varphi_{h}\left(\frac{\tilde{w}_{r, C}}{2}, t\right)=\frac{1}{2}\left(1-\tanh \left(\left(\frac{2}{\sqrt{\pi}}\right)\left(\frac{\frac{\tilde{w}_{r, C}}{2}-z_{c 0}\left(1-e^{\frac{-t}{\tau}}\right)-z_{c}}{z_{c}+\sqrt{\frac{z(t)}{z_{c 1}}}}\right)\right)\right)
$$

\section{Crack-plane constitutive model}

In the present work, different constitutive models -that are coupled through the equilibrium of stresses and the crack initiation criterion [58] are employed for the continuum and the discrete cracks. The continuum is assumed to be linear elastic, whilst the discontinuity is assumed to follow the elastic-damage-healing cohesive crack constitutive relationship given in Eq. (21).

\section{Damage-only model}

The damage variable $(\tilde{\omega})$ is a function of the damage evolution parameter $(\tilde{\zeta})$, as follows:

$$
\tilde{\omega}=1-\frac{u_{t}}{\tilde{\zeta}} \cdot e^{-c_{1} \frac{\tilde{\zeta}-u_{t}}{u_{m}-u_{t}}}
$$

where $u_{t}=f_{t} / K$ where $f_{t}$ is the tensile strength of the material, $K=E / h_{c p}$ is the crackplane stiffness, $E$ is Young's modulus of the continuum material (also the crack-plane band of elastic material), $h_{c p}$ is the assumed thickness of the crack-plane (taken as $10 \mathrm{~mm}$ u.n.o.), $u_{m}$ is the relative-displacement at the effective end of the softening curve (taken as $0.2 \mathrm{~mm}$ u.n.o.) and $c_{1}=5$ is a softening constant.

The evolution of $\tilde{\zeta}$ is governed by the following damage function:

$$
\varphi_{d}(\tilde{u}, \tilde{\zeta})=\zeta_{e q}(\tilde{u})-\tilde{\zeta}
$$

in $\quad$ which $\zeta_{e q}(\tilde{u})=\frac{\tilde{u}_{1}}{2}\left[1+\left(\frac{\mu}{\gamma}\right)^{2}\right]+\frac{1}{2 \gamma^{2}} \sqrt{\left(\gamma^{2}-\mu^{2}\right)^{2} \tilde{u}_{1}^{2}+4 \gamma^{2}\left(\tilde{u}_{2}^{2}+\tilde{u}_{3}^{2}\right)}$ with the standard loading/unloading conditions $\dot{Z} \geq 0 ; \phi_{d} \leq 0 ; \dot{\mathrm{Z}} \cdot \phi_{d}=0 ; \dot{\mathrm{Z}} \dot{\phi}_{d}=0 \forall \phi_{d}=0$.

\section{Damage-healing model}

The damage component of the model is unchanged from that described by Eqs. (37) and (38). Healing depends on the flow and curing of the healing agent, as explained in the previous section.

To allow for re-damage and re-healing, the evolution of the healing variable, found in the elastic-damage-healing cohesive crack constitutive relationship given in Eq. (21), is to be based on the following;

$$
\tilde{h}=\tilde{h} \cdot e^{-\Delta t / \tau_{h}}+a \cdot\left(1-e^{-\Delta t / \tau_{h}}\right)
$$

in which the relative area $(a)$ of the crack exposed to healing agent is given by; 


$$
a=a+\Delta a_{c}-\Delta a_{\text {redam }}+\Delta a_{r e c}
$$

in which $\Delta a_{c}$ is the incremental area of virgin filled crack, $\Delta a_{\text {redam }}$ is the incremental area of re-damaged material and $\Delta a_{r e c}$ is the incremental area of re-filled cracks.

An important aspect of the damage-healing model is that an increment of healing causes no change in mechanical energy when it takes place in a static crack. This is equivalent to saying that healing agent cures in a stress-free state. Applying this principle to Eq. (21) for a healing update $(\Delta h)$, with $\tilde{\mathbf{u}}$ remaining constant, leads to the following expression:

$$
(\tilde{h}+\Delta \tilde{h}) \tilde{\mathbf{k}}^{h e}\left(\tilde{\mathbf{u}}-\tilde{\mathbf{u}}_{h}-\Delta \tilde{\mathbf{u}}_{h}\right)-\tilde{h} \tilde{\mathbf{k}}^{h e}\left(\tilde{\mathbf{u}}-\tilde{\mathbf{u}}_{h}\right)=\mathbf{0}
$$

from which the update $\left(\Delta \tilde{\mathbf{u}}_{h}\right)$ may be derived to be:

$$
\Delta \tilde{\mathbf{u}}_{h}=\frac{\Delta \tilde{h}}{(\tilde{h}+\Delta \tilde{h})}\left(\tilde{\mathbf{u}}-\tilde{\mathbf{u}}_{h}\right)
$$

\section{Example problems}

In this section, example problems are presented to demonstrate the performance of the model. Crack continuity was ensured using the algorithm of Alfaiate et al. [49, 79] whilst U-turns in the crack path were prevented using the approach of Cervera et al. [80]. The latter two examples concern self-healing concrete specimens with embedded vascular networks. Depending upon the configuration of the channels, such systems can require a three-dimensional approach, since the transport of the healing agent may not be uniform across the width of the specimen. However, for the present examples, the authors believe that two-dimensional idealisations are adequate. This is justified by the fact that in the direct tension example, experimental observations showed that the healing agent had spread uniformly across the width of the crack [81, 82]. Whilst in the L-shaped specimen example, the embedded channels are hypothetical and it is assumed that there are sufficient channels for the healing agent flow front to be effectively uniform across the specimen. The model parameters employed for the flow model can be seen in the Appendix.

\section{Convergence test}

This example considers a hypothetical prismatic singly notched specimen that is loaded in tension, as illustrated in Fig. 3. In this test it was assumed that the rate of transport of the healing agent in the crack was much faster than the rate of loading such that the crack was filled instantaneously with healing agent. The finite element meshes used in the analysis can be seen in Fig. 4, whilst the model parameters are given in Table 1 . The number of elements used ranged from 36 for Mesh1 to 1296 for Mesh4, the time step employed was $0.5 \mathrm{~s}$. A convergence tolerance of $0.01 \%$ for the out of balance force and $L^{2}$ iterative displacement norms was employed in this example and an average of 5 Newton iterations were required per time step. 
The load response curves from the simulations are given in Fig. 5. It can be seen from the figure that there is a significant difference in the load-response when healing is considered, in addition to there being a greater difference in the responses predicted for each of the meshes employed. To analyse the mesh convergence, the $L^{2}$ -error norm of the response curves was employed:

$$
\frac{\left\|\boldsymbol{P}^{r}-\boldsymbol{P}\right\|_{L^{2}}}{\left\|\boldsymbol{P}^{r}\right\|_{L^{2}}}=\sqrt{\frac{\int\left(\boldsymbol{P}^{r}-\boldsymbol{P}\right)\left(\boldsymbol{P}^{r}-\boldsymbol{P}\right) d s}{\int\left(\boldsymbol{P}^{r}\right)\left(\boldsymbol{P}^{r}\right) d s}}
$$

where $\boldsymbol{P}$ and $\boldsymbol{P}^{r}$ are the vectors of loads found in the response curves for a given solution and the reference solution respectively and the integral is taken over the response curve.

In the absence of an analytical solution to the problem, the solutions obtained with Mesh4 for the damage only and damage-healing cases were taken as the reference solutions. The results of the convergence study can be seen in Fig. 6, where the error norm is plotted against the element size, $h_{e}$. The figure shows that the characteristics of the convergence behaviour of the model are the same for the damage only and damage-healing case, but that the error is larger when healing is considered.

\section{Direct tension test}

This example considers a set of direct tension tests on doubly notched concrete specimens (illustrated in Fig. 7), which contained embedded healing channels that were filled with cyanoacrylate (CA), presented in Selvarajoo [81] and Selvarajoo et al. [82]. The tests involved loading the specimen until a macro crack had formed and then opened to a given crack mouth opening displacement (CMOD). The CA supply was then released whilst the crack was then held at the CMOD value for a selected period of time. At the end of this 'healing period', the specimen was loaded to until the CMOD clip gauge reached $0.3 \mathrm{~mm}$, after which the specimens were unloaded. The test series considered two different crack openings and fixed healing periods of $0 \mathrm{~s}, 60 \mathrm{~s}$ and $600 \mathrm{~s}$ (and $1200 \mathrm{~s}$ for the $0.2 \mathrm{~mm}$ opening). The test set up can be seen in Fig. 7. The finite element meshes used in the analyses can be seen in Fig. 8. The model parameters are given in Table 2, where the subscript $h$ indicates that these parameters are for the healed material. The

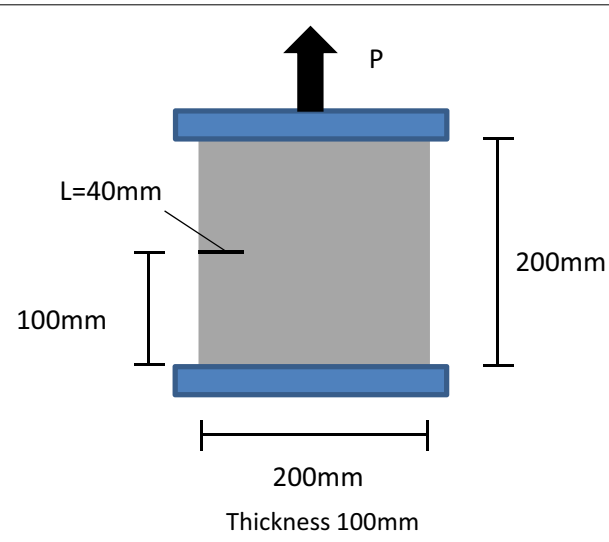

Fig. 3 Schematic of the convergence test set up 

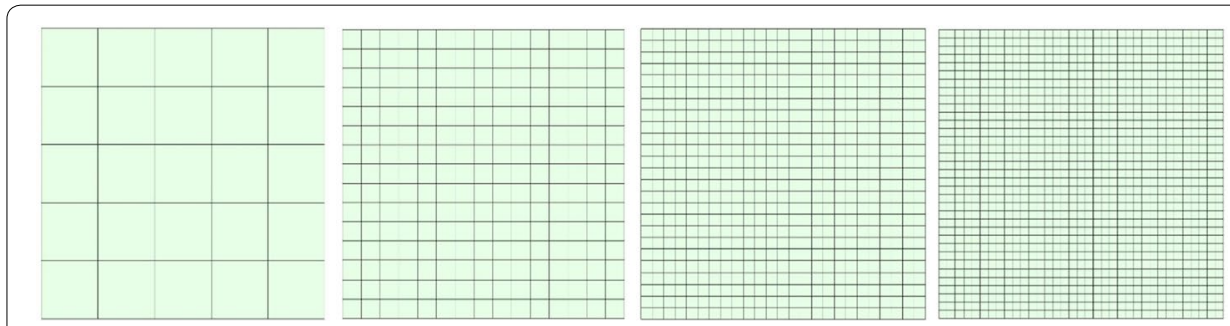

Fig. 4 Finite element meshes used, namely Mesh1 (left), Mesh2 (centre left), Mesh3 (centre right) and Mesh4 (right)

Table 1 Model parameters convergence test

\begin{tabular}{llll}
\hline Parameter & Value & Parameter & Value \\
\hline$E\left(\mathrm{~N} / \mathrm{mm}^{2}\right)$ & 30,000 & $f_{\text {th }}\left(\mathrm{N} / \mathrm{mm}^{2}\right)$ & 3.00 \\
$\nu(-)$ & 0.2 & $\varepsilon_{\text {emh }}(\mathrm{mm})$ & 0.4 \\
$f_{t}\left(\mathrm{~N} / \mathrm{mm}^{2}\right)$ & 3.00 & $z_{c 0}(\mathrm{~mm})$ & 0.16 \\
$\varepsilon_{e m}(\mathrm{~mm})$ & 0.15 & $\tau(\mathrm{s})$ & 120 \\
$h_{b}(\mathrm{~mm})$ & 4.0 & $z_{c 1}\left(\mathrm{~mm} / \mathrm{mm}^{2}\right)$ & 25 \\
$E_{h}\left(\mathrm{~N} / \mathrm{mm}^{2}\right)$ & 30,000 & $z_{c}(\mathrm{~mm})$ & $1 \times 10^{-5}$ \\
$\nu_{h}(-)$ & 0.2 & & \\
\hline
\end{tabular}
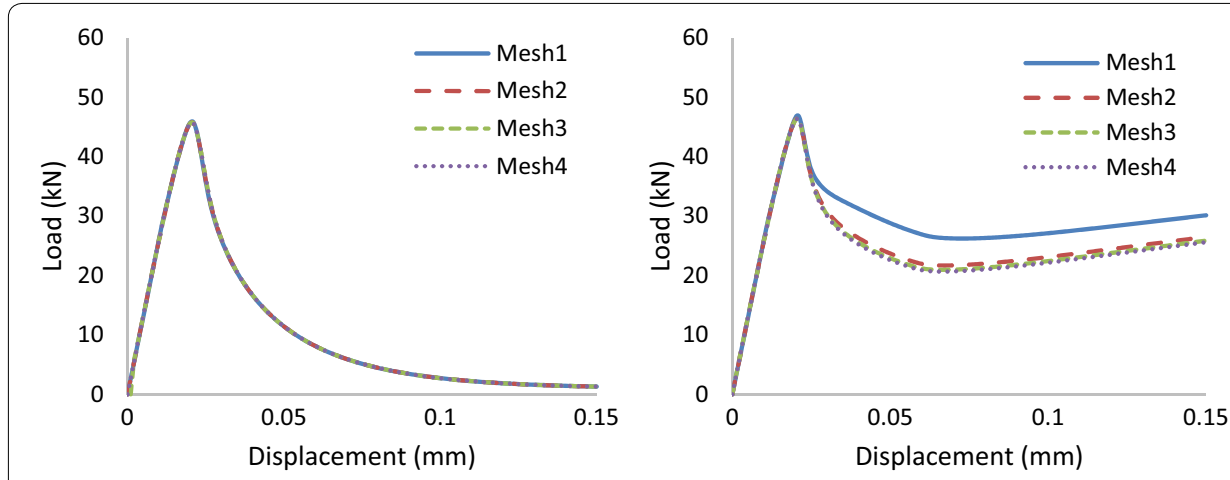

Fig. 5 Load displacement curve damage only (left) and damage-healing (right)

number of elements was 144 and 506 for Mesh1 and Mesh2 respectively, whilst the time step size used was $1 \mathrm{~s}$. A convergence tolerance of $0.01 \%$ for the out of balance force and $L^{2}$ iterative displacement norms was employed in this example and an average of 4 Newton iterations were required per time step.

It can be noted that in this example, following experimental observations, the degree of healing was limited to 0.85 . This is due to the fact that in some areas of the crack, the healing agent was in constant flux and as such did not stabilise and cure [82].

The comparison of the numerical simulations with the experimental data is given in Fig. 9. It can be seen from the figure that the numerical predictions accurately capture the experimental behaviour for both the $0.1 \mathrm{~mm}$ and $0.2 \mathrm{~mm}$ cases. The comparison of 


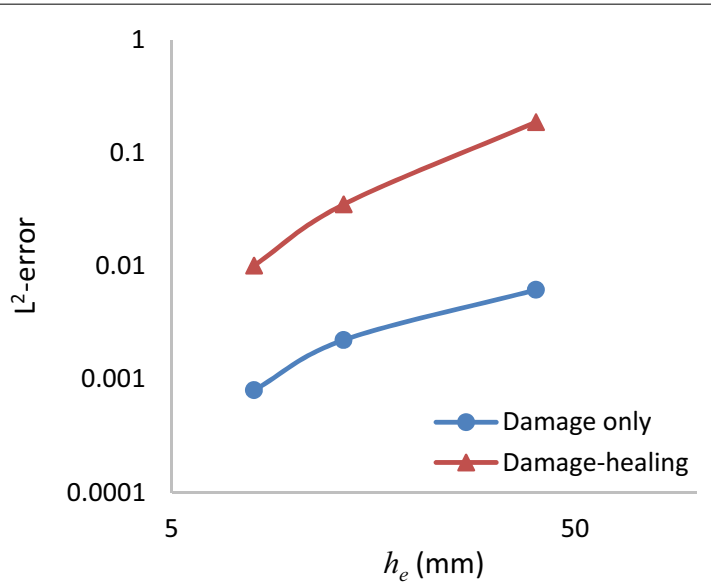

Fig. 6 Convergence in terms of the $L^{2}$-error norm of the response curves

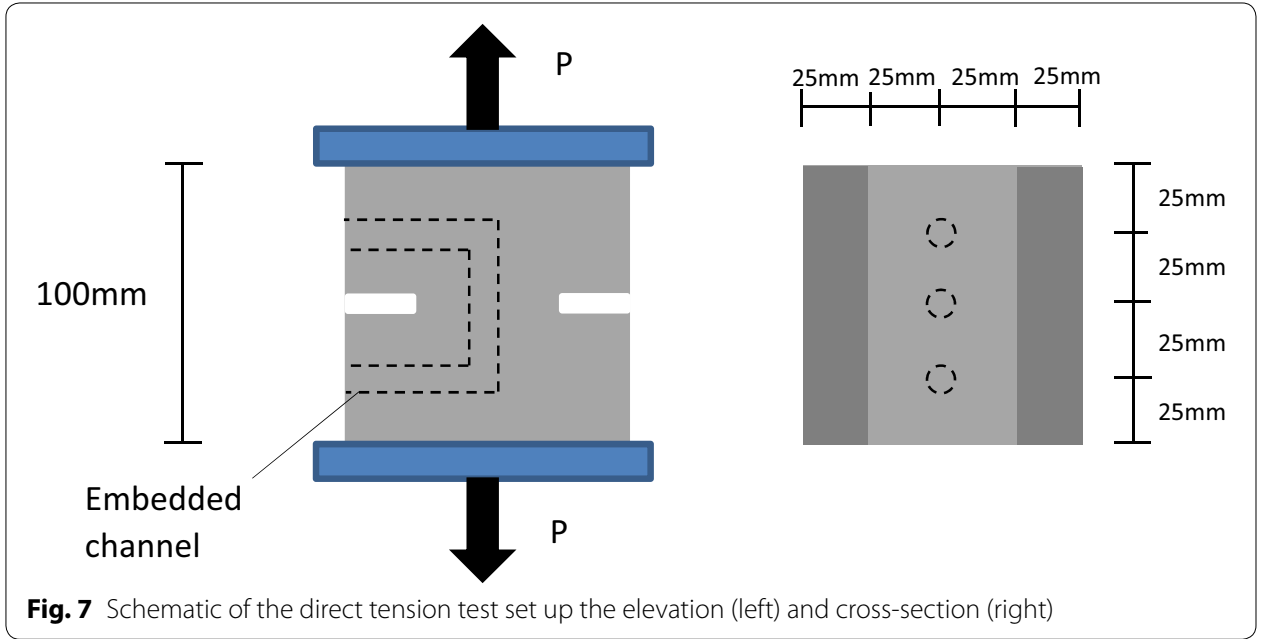

the predicted and experimentally observed crack pattern can be seen in Fig. 10. It can be seen from the figure that there is a generally good match, although the experimental crack path is more tortuous. In this example, cracks propagated from the notches to the centre of the specimen, where they coalesced into one crack that crossed the centre of the specimen. The crack was fully formed prior to the release of healing agent in both cases and, as a result of this, the healing had no effect on the crack pattern. The numerical response curves for the two meshes are coincident, indicating mesh convergence.

\section{L-shaped specimen test}

This next example concerns the L-shaped specimen presented in Winkler et al. [83]. The test considered both plain and reinforced concrete specimens and both the load displacement curves and crack patterns were recorded. In the present study, the plain specimens were considered. To investigate the effect of healing, two hypothetical embedded channels filled with CA and placed vertically at a distances of $175 \mathrm{~mm}$ and $115 \mathrm{~mm}$ from the right-hand-side of the specimen were considered. The location of these hypothetical 

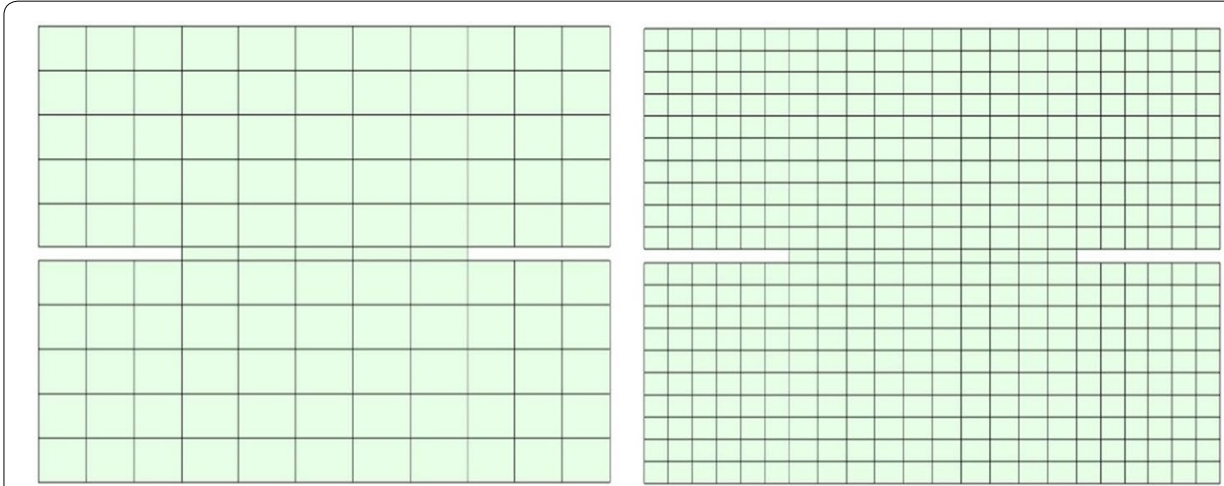

Fig. 8 Finite element meshes used, namely Mesh1 (left) and Mesh2 (right)

Table 2 Model parameters direct tension test

\begin{tabular}{llll}
\hline Parameter & Value & Parameter & Value \\
\hline$E\left(\mathrm{~N} / \mathrm{mm}^{2}\right)$ & 30,000 & $f_{\text {th }}\left(\mathrm{N} / \mathrm{mm}^{2}\right)$ & 3.75 \\
$\nu(-)$ & 0.2 & $\varepsilon_{\text {emh }}(\mathrm{mm})$ & 0.4 \\
$f_{t}\left(\mathrm{~N} / \mathrm{mm}^{2}\right)$ & 3.75 & $z_{c 0}(\mathrm{~mm})$ & 0.16 \\
$\varepsilon_{e m}(\mathrm{~mm})$ & 0.15 & $\tau(\mathrm{s})$ & 120 \\
$h_{b}(\mathrm{~mm})$ & 4.0 & $z_{c 1}\left(\mathrm{~mm} / \mathrm{mm}^{2}\right)$ & 25 \\
$E_{h}\left(\mathrm{~N} / \mathrm{mm}^{2}\right)$ & 30,000 & $z_{c}(\mathrm{~mm})$ & $1 \times 10^{-5}$ \\
$\nu_{h}(-)$ & 0.2 & & \\
\hline
\end{tabular}

channels can be seen in Fig. 11. The finite element meshes used in the analyses can be seen in Fig. 12, whilst the model parameters are given in Table 3. The meshes include two structured meshes and one unstructured mesh that contains distorted elements. The number of elements was 770, 1158 and 1976 for Mesh1, Mesh2 and Mesh3 respectively, whilst the time step size used was $10 \mathrm{~s}$. A convergence tolerance of $0.01 \%$ for the out of balance force and $L^{2}$ iterative displacement norms was employed in this example and an average of 5 Newton iterations were required per time step.

The comparison of the numerical simulations with the experimental data can be seen in Figs. 13 and 14, whilst contour plots of the degree of healing in the crack at four points in the test (indicated by markers a-d in Fig. 13) is given in Fig. 15. It may be seen from the figures that the numerical predictions for the damage only case are able to accurately capture the experimental behaviour in terms of both the load-displacement curve and the predicted crack patterns. It is interesting to note the healing has a significant effect on the load-displacement curve, but that the predicted crack pattern remains unchanged. A key feature of the post peak response for the healing case is the two distinct post healed peak loads, each of which follows the release of CA from one of the embedded channels. The contour plots show that the degree of healing is greater than $80 \%$ throughout the crack at the first healed peak load, and that by the end of the test almost all of the healed material has been completely redamaged. The numerical response curves for the three meshes are in very close agreement, indicating mesh convergence. In addition to this, the predicted crack patterns are also in good agreement. 

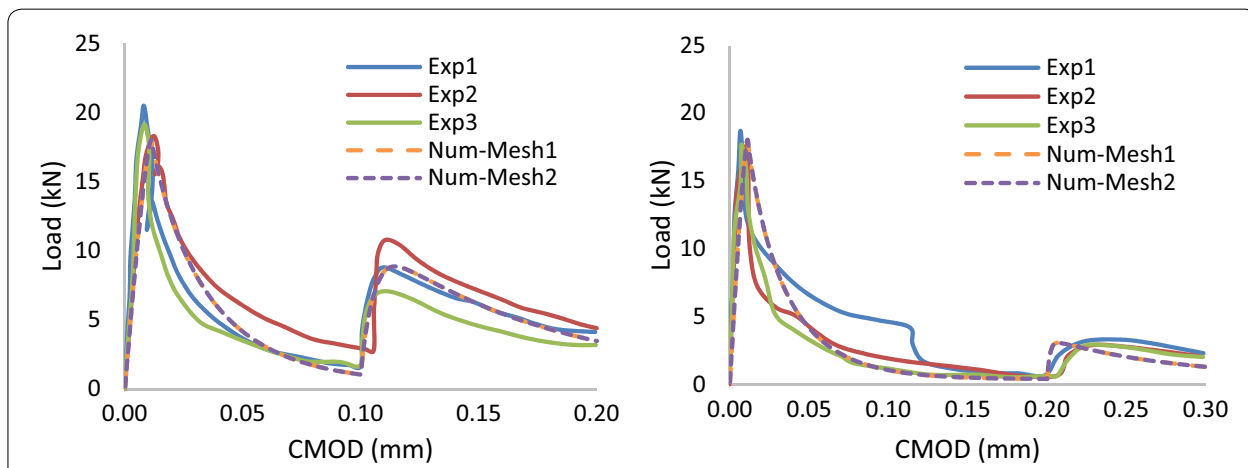

Fig. 9 Comparison of load-CMOD curve with experimental data for a fixed healing period of $60 \mathrm{~s}$ and crack widths of $0.1 \mathrm{~mm}$ (left) and $0.2 \mathrm{~mm}$ (right)

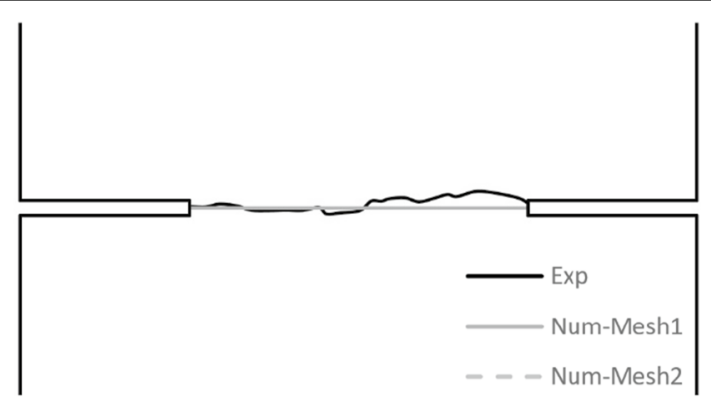

Fig. 10 Comparison of numerical and experimentally observed crack pattern

\section{Conclusions}

A novel specialised element for simulating self-healing in quasi-brittle materials was presented. The following conclusions can be drawn from this work:

- The introduction of the healing variables at the element level ensures consistency with the internal degrees of freedom associated with the crack.

- The equilibrium condition over the crack plane can be satisfied in a weak sense using the virtual work associated with the internal degrees of freedom.

- The proposed element allows for different healing mechanisms and is readily coupled to a transport model that could be used to calculate the amount of available healing agent at a damage site.

- The proposed element allows for overlapping cycles of damage and healing when combined with a suitable crack-plane model. 


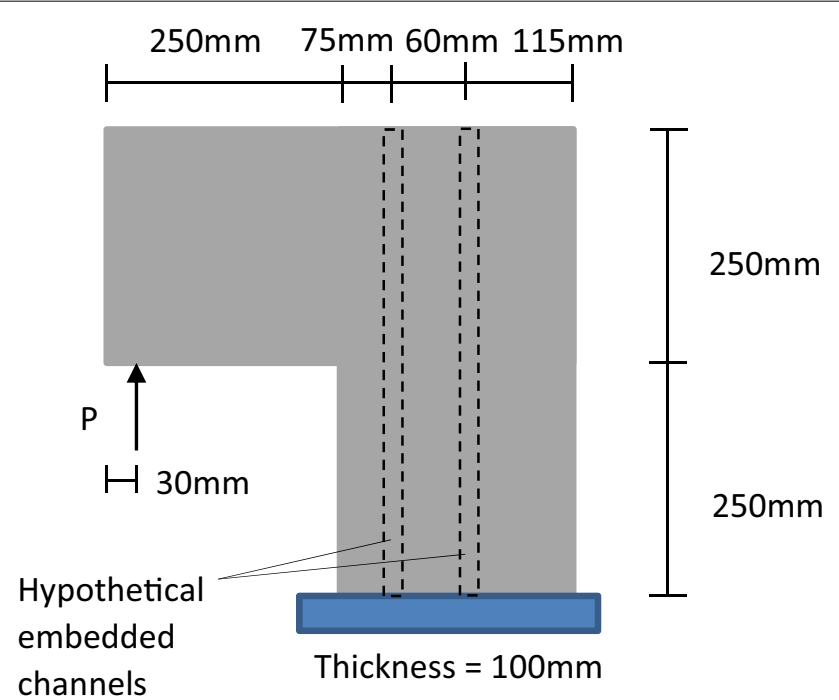

Fig. 11 Schematic of the $L$ beam test set up

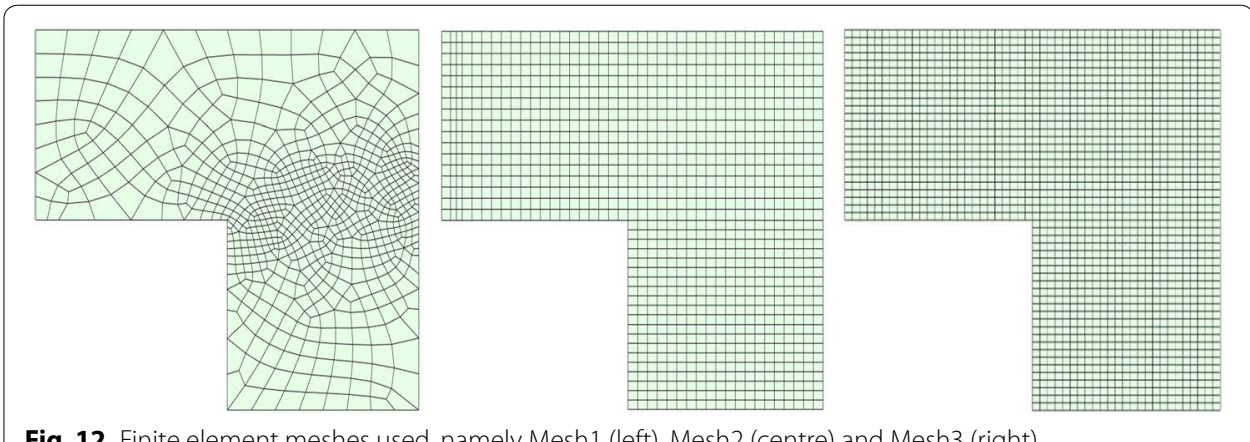

Fig. 12 Finite element meshes used, namely Mesh1 (left), Mesh2 (centre) and Mesh3 (right)

Table 3 Model parameters L-shaped specimen test

\begin{tabular}{llll}
\hline Parameter & Value & Parameter & Value \\
\hline$E\left(\mathrm{~N} / \mathrm{mm}^{2}\right)$ & 25,850 & $f_{\text {th }}\left(\mathrm{N} / \mathrm{mm}^{2}\right)$ & 6.75 \\
$\nu(-)$ & 0.18 & $\varepsilon_{\text {emh }}(\mathrm{mm})$ & 0.4 \\
$f_{t}\left(\mathrm{~N} / \mathrm{mm}^{2}\right)$ & 2.7 & $z_{c 0}(\mathrm{~mm})$ & 0.26 \\
$\varepsilon_{e m}(\mathrm{~mm})$ & 0.225 & $\tau(\mathrm{s})$ & 30 \\
$h_{b}(\mathrm{~mm})$ & 4.0 & $z_{c 1}\left(\mathrm{~mm} / \mathrm{mm}^{2}\right)$ & 25 \\
$E_{h}\left(\mathrm{~N} / \mathrm{mm}^{2}\right)$ & 30,000 & $z_{c}(\mathrm{~mm})$ & $1 \times 10^{-5}$ \\
$\nu_{h}(-)$ & 0.2 & & \\
\hline
\end{tabular}




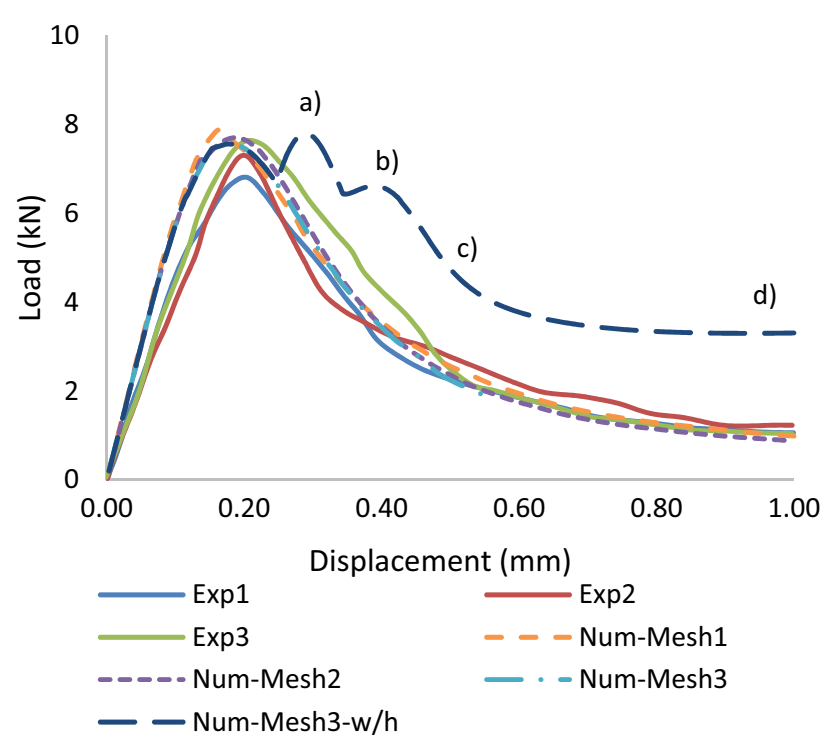

Fig. 13 Comparison of load-displacement curve with experimental data

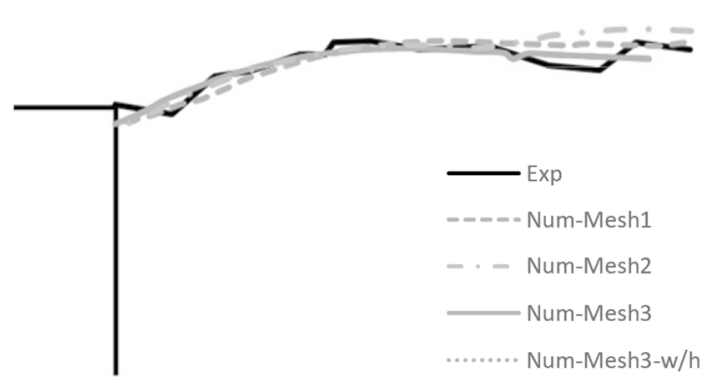

Fig. 14 Comparison of numerical and experimentally observed crack pattern

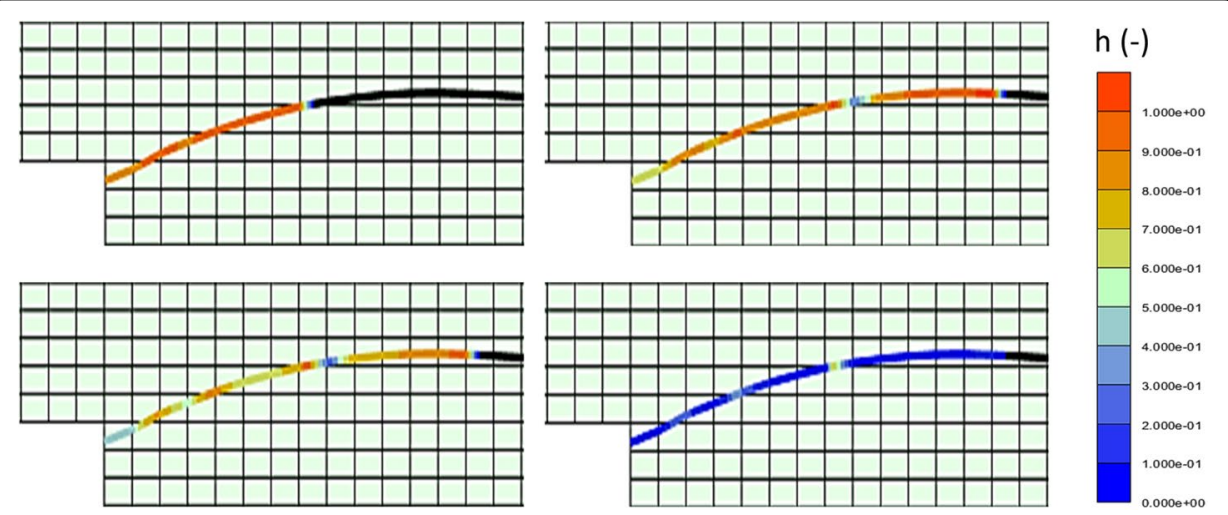

Fig. 15 Contour plots of degree of healing at a (top left), $\mathbf{b}$ (top right), $\mathbf{c}$ (bottom left) and $\mathbf{d}$ (bottom right) 
- The new finite element model is able to accurately capture the damage-healing behaviour of a vascular self-healing cementitious material system in terms of both the mechanical response and the observed crack patterns, as demonstrated with the validation examples.

Acknowledgements

Not applicable.

Authors' contributions

BLF, PBV, ICM, WFA, ADJ have contributed to this study. All authors read and approved the final manuscript.

Funding

This work is supported by UKRI-EPSRC Grant No. EP/P02081X/1, Resilient Materials 4 Life, RM4L and Grant No. EP/

P033792/1, Two-scale numerical simulations for fibre reinforced cementitious composites.

Availability of data and materials

Information on the data underpinning the results presented here, including how to access them, can be found in the Cardiff University data catalogue at (http://doi.org/ https://doi.org/10.17035/d.2020.0099148076).

Competing interests

The authors declare that they have no competing interests.

Author details

${ }^{1}$ School of Engineering, Cardiff University, Cardiff CF24 3AA, UK. ${ }^{2}$ Elmergib University, Alkhoms, Libya.

\section{Appendix}

\section{Healing agent transport and curing model parameters}

The parameters used for the flow model can be seen in Table 4. In the L-shaped specimen test the flow is initiated once the crack width at the location of the channel reaches a critical value $\left(w_{\text {crit }}\right)$.

Table 4 Flow model parameters

\begin{tabular}{llll}
\hline Parameter & Value & Parameter & Value \\
\hline$\theta_{s}(\mathrm{rad})$ & 0.1754 & $\beta_{s}(-)$ & 0 \\
$\gamma(\mathrm{N} / \mathrm{m})$ & 0.033 & $c_{1}(-)$ & 1.325 \\
$\mu\left(\mathrm{Ns} / \mathrm{m}^{2}\right)$ & 0.004 & $c_{2}(-)$ & 0.35 \\
$\rho\left(\mathrm{kg} / \mathrm{m}^{3}\right)$ & 1060 & $W_{c r i t}(\mathrm{~mm})$ & 0.025 \\
$\beta_{m}\left(\mathrm{Ns} / \mathrm{m}^{2}\right)$ & 0 & $P_{a p p}{ }^{\mathrm{a}}\left(\mathrm{N} / \mathrm{m}^{2}\right)$ & 50,000 \\
$\beta_{w}\left(\mathrm{~m}^{3} / \mathrm{Ns}\right)$ & 0 & $P_{a p p}{ }^{\mathrm{b}}\left(\mathrm{N} / \mathrm{m}^{2}\right)$ & 500,000 \\
\hline
\end{tabular}

a Direct tension test

b L-shaped specimen test 
Received: 3 February 2020 Accepted: 11 June 2020

Published online: 02 July 2020

\section{References}

1. Baroghel-Bouny V, Nguyen TQ, Dangla P. Assessment and prediction of RC structure service life by means of durability indicators and physical/chemical models. Cement Concr Compos. 2009;31:522-34.

2. Schimmel EC, Remmers J. Development of a constitutive model for self-healing materials. Delft Aerospace Computational Science, Report DACS-06-003 2006.

3. Remmers J, de Borst R. Numerical modelling of self-healing mechanisms. In Self Healing Materials Springer. 2008. 365-380.

4. Abu Al-Rub R, Darabi MK, Little DN, Masad EA. A micro-damage healing model that improves the prediction of fatigue life in asphalt mixes. Int J Eng Sci. 2010;48:966-90.

5. Voyiadjis GZ, Shojaei A, Li G. A thermodynamic consistent damage and healing model for self healing materials. Int J Plast. 2011;27:1025-44.

6. Huang H, Ye G. Simulation of self-healing by further hydration in cementitious materials. Cement Concr Compos. 2012;34:460-7.

7. Mergheim J, Steinmann P. Phenomenological modelling of self-healing polymers based on integrated healing agents. Comput Mech. 2013;52:681-92.

8. Aliko-Benitez A, Doblare M, Sanz-Herrera JA. Chemical-diffusive modeling of the self-healing behaviour in concrete. Int J Solids Struct. 2015;69-70:392-402.

9. Chitez AS, Jefferson AD. A coupled thermo-hygro-chemical model for characterising autogenous healing in ordinary cementitious materials. Cem Concr Res. 2016;88:184-97.

10. Caggiano A, Etse G, Ferrara L, Krelani V. Zero-thickness interface constitutive theory for concrete self-healing effects. Comput Struct. 2017;186:22-34.

11. Davies R, Jefferson AD. Micromechanical modelling of self-healing cementitious materials. Int J Solids Struct. 2017:113-114:180-91.

12. Gilabert FA, Garoz D, Van Paepegem W. Macro- and micro-modeling of crack propagation in encapsulationbased self-healing materials: application of xfem and cohesive surface techniques. Mater Des. 2017:130:459-78.

13. Zhou S, Zhu H, Ju JW, Yan Z, Chen Q. Modeling microcapsule-enabled self-healing cementitious composite materials using discrete element method. Int J Damage Mech. 2017;26:340-57.

14. Di Luzio G, Ferrara L, Krelani V. Numerical modeling of mechanical regain due to self-healing in cement based composites. Cement Concr Compos. 2018:86:190-205.

15. Oucif C, Voyiadjis GZ, RabczukT. Modeling of damage-healing and nonlinear self-healing concrete behaviour: application to coupled and uncoupled self-healing mechanisms. Theoret Appl Fract Mech. 2018;96:216-30.

16. Ponnusami SA, Krishnasamy J, Turteltaub S, van der Zwaag S. A cohesive-zone crack healing model for selfhealing materials. Int J Solids Struct. 2018;134:249-63.

17. Zhang Y, Zhuang $X$. A softening-healing law for self-healing quasi-brittle materials: analysing with strong discontinuity embedded approach. Eng Fract Mech. 2018;192:290-306.

18. Sanz-Herrera JA, Aliko-Benitez A, Fadrique-Contreras AM. Numerical investigation of the coupled mechanical behaviour of self-healing materials under cyclic loading. Int J Solids Struct. 2019;160:232-46.

19. Freeman BL, Jefferson AD. The simulation of transport processes in cementitious materials with embedded healing systems. Int J Numer Anal Meth Geomech. 2020;44:293-326.

20. Jefferson AD, Javierre E, Freeman B, Zaoui A, Koenders E, Ferrara L. Research progress on numerical models for self-healing cementitious materials. Adv Mater Interf. 2018;5:1701378.

21. Rots JG. Smeared and discrete representations of localized fracture. Int J Fract. 1991;51 (1):45-59.

22. Noghabai K. Discrete versus smeared versus element-embedded crack models on ring problem. J Eng Mech. 1999;125(3):307-15.

23. de Borst R, Remmers JJC, Needleman A, Abellan M-A. Discrete vs smeared crack models for concrete fracture: bridging the gap. Int J Numer Anal Meth Geomech. 2004;28:583-607.

24. Dias-da-Costa D, Cervenka V, Graça-e-Costa R. Model uncertainty in discrete and smeared crack prediction in RC beams under flexural loads. Eng Fract Mech. 2018;199:532-43.

25. Belytschko T, Black T. Elastic crack growth in finite elements with minimal remeshing. Int J Numer Meth Eng. 1999:45:601-20

26. Moës N, Dolbow J, Belytschko T. A finite element method for crack growth without remeshing. Int J Numer Meth Eng. 1999;46:131-50.

27. Duarte CAM, Babuška I, Oden JT. Generalized finite element methods for three-dimensional structural mechanics problems. Comput Struct. 2000;77:215-32.

28. Song JH, Areias PMA, Belytschko T. A method for dynamic crack and shear band propagation with phantom nodes. Int J Numer Meth Eng. 2006;67:868-93.

29. Melenk JM, Babuška I. The partition of unity finite element method: basic theory and applications. Comput Methods Appl Mech Eng. 1996;139:289-314.

30. Bordas S, Nguyen PV, Dunant C, Guidoum A, Nguyen-Dang H. An extended finite element library. Int J Numer Meth Eng. 2007;71:703-32.

31. Karihallo BL, Xiao QZ. Modelling of stationary and growing cracks in FE framework without remeshing: a state-ofthe-art review. Comput Struct. 2003:81:119-29.

32. Fries TP, Belytschko T. The extended/generalized finite element method: an overview of the method and its applications. Int J Numer Meth Eng. 2010:84:253-304. 
33. YuT, Bui TQ. Numerical simulation of 2-D weak and strong discontinuities by a novel approach based on XFEM with local mesh refinement. Comput Struct. 2018;196:112-33.

34. Strouboulis T, Babuška I, Copps K. The design and analysis of the generalized finite element method. Comput Methods Appl Mech Eng. 2000;181:43-69.

35. Strouboulis T, Copps A, Babuška I. The generalized finite element method: an example of its implementation and illustration of its performance. Int J Numer Methods Eng. 2000;47:1401-17.

36. Strouboulis T, Copps K, Babuška I. The generalized finite element method. Comput Methods Appl Mech Eng. 2001;190:4081-193.

37. Babuška I, Caloz G, Osborn JE. Special finite element methods for a class of second order elliptic problems with rough coefficients. SIAM J Numer Anal. 1994;31:745-981.

38. Babuška I, Melenk JM. The partition of unity method. Int J Numer Meth Eng. 1997:40:727-58.

39. Oden JT, Duarte CAM, Zienkiewicz OC. A new cloud-based hp finite element method. Comput Methods Appl Mech Eng. 1998;153:117-26.

40. Belytschko T, Gracie R, Ventura G. A review of extended/generalized finite element methods for material modelling. Modell Simul Mater Sci Eng. 2009;17:043001.

41. Hansbo A, Hansbo P. An unfitted finite element method, based on Nitche's method, for elliptic interface problems. Comput Methods Appl Mech Eng. 2002;191:5537-52.

42. Hansbo A, Hansbo P. A finite element method for the simulation of strong and weak discontinuities in solid mechanics. Comput Methods Appl Mech Eng. 2004;193:3523-40.

43. Burman E, Claus S, Hansbo P, Larson MG, Massing A. CutFEM: discretizing geometry and partial differential equations. Int J Numer Meth Eng. 2015;104:472-501.

44. Schott B, Wall WA. A new face-oriented stabilized XFEM approach for 2D and 3D incompressible Navier-Stokes equations. Comput Methods Appl Mech Eng. 2014;276:233-65.

45. Hansbo P, Larson MG, Massing A. A stabilized cut finite element method for the Darcy problem on surfaces. Comput Methods Appl Mech Eng. 2017;326:298-318.

46. Claus S, Kerfriden P. A stable and optimally convergent LaTIn-CutFEM algorithm for multiple unilateral contact problems. Int J Numer Meth Eng. 2017;113:938-66.

47. Burman E, Elfverson D, Hansbo P, Larson MG, Larsson K. Shape optimisation using the cut finite element method. Comput Methods Appl Mech Eng. 2018;328:242-61.

48. Claus S, Kerfriden P. A CutFEM method for two-phase flow problems. Comput Methods Appl Mech Eng 2019:348:185-206.

49. Alfaiate J, Simone A, Sluys LJ. Non-homogeneous displacement jumps in strong embedded discontinuities. Int J Solids Struct. 2003;40(21):5799-817. https://doi.org/10.1016/S0020-7683(03)00372-X.

50. Oliver J, Huespe AE, Sanchez PJ. A comparative study on finite elements for capturing strong discontinuities: E-FEM vs X-FEM. Comput Methods Appl Mech Eng. 2006;195:4732-52.

51. Ortiz M, Leroy Y, Needleman A. A finite element method for localized failure analysis. Comput Methods Appl Mech Eng. 1987;61:189-214.

52. Dvorkin EN, Cuitino AM, Gioia G. Finite elements with displacement interpolated embedded localization lines insensitive to mesh size and distortions. Int J Numer Meth Eng. 1990;30:541-64.

53. Simo JC, Oliver J, Armero F. An analysis of strong discontinuities induced by strain softening in rate-independent inelastic solids. Comput Mech. 1993;12:277-96.

54. Lotfi HR, Shing PB. Embedded representation of fracture in concrete with mixed finite elements. Int J Numer Meth Eng. 1995;38:1307-25.

55. Oliver J. Modelling strong discontinuities in solid mechanics via strain softening constitutive equations. Part 1: fundamentals. Int J Numer Methods Eng. 1996;39:3575-600.

56. Oliver J. Modelling strong discontinuities in solid mechanics via strain softening constitutive equations. Part 2: numerical simulation. Int J Numer Methods Eng. 1996;39:3601-23.

57. Wells GN, Sluys LJ. Application of embedded discontinuities for softening solids. Eng Fract Mech. 2000;65:263-81.

58. Dias-da-Costa D, Alfaiate J, Sluys LJ, Julio E. A discrete strong discontinuity approach. Eng Fract Mech. 2009;76:1176-201.

59. Dias-da-Costa D, Alfaiate J, Sluys LJ, Julio E. Towards a generalization of a discrete discontinuity approach. Comput Methods Appl Mech Eng. 2009;198:3670-81.

60. Dias-da-Costa D, Alfaiate J, Sluys LJ, Areias P, Julio E. An embedded formulation with conforming finite elements to capture strong discontinuities. Int J Numer Meth Eng. 2013;93:224-44.

61. Linder C, Armero F. Finite elements with embedded branching. Finite Elem Anal Des. 2009:45:280-93.

62. Armero F, Linder C. Numerical simulation of dynamic fracture using finite elements with embedded discontinuities. Int J Fract. 2009;160:119-41.

63. Djuc A, Brank B, Ibrahimbegovic A. Stress-hybrid quadrilateral finite element with embedded strong discontinuity for failure analysis of plane stress solids. Int J Numer Meth Eng. 2013;94:1075-98.

64. Saksala T, Brancherie D, Harari I, Ibrahimbegovic A. Combined continuum damage-embedded discontinuity model for explicit dynamic fracture analyses of quasi-brittle materials. Int J Numer Meth Eng. 2015;101:230-50.

65. Saksala T, Brancherie D, Ibrahimbegovic A. Numerical modeling of dynamic rock fracture with a combined 3D continuum viscodamage-embedded discontinuity model. Int J Numer Anal Meth Geomech. 2016;40:1339-57.

66. Lu M, Zhang H, Zheng Y, Zhang L. A multiscale finite element method with embedded strong discontinuity model for the simulation of cohesive cracks in solids. Comput Methods Appl Mech Eng. 2016;311:576-98.

67. Lu M, Zhang H, Zheng Y, Zhang L. A multiscale finite element method for the localization analysis of homogeneous and heterogeneous saturated porous media with embedded strong discontinuity model. Int J Numer Meth Eng. 2017:112:1439-72.

68. Jirasek M. Comparative study on finite elements with embedded discontinuities. Comput Methods Appl Mech Eng 2000;188:307-30. 
69. Hou TY, Wu XH. A multiscale finite element method for elliptic problems in composite materials and porous media. J Comput Phys. 1997;134(1):169-89.

70. Jefferson AD, Selvarajoo T, Freeman BL, Davies R. An experimental and numerical study on vascular self-healing cementitious materials. MATEC Web Conf. Concrete solutions 2019-7th international conference on concrete repair. 2019.

71. Jefferson AD, Mihai IC, Tenchev R, Alnaas WF, Cole G, Lyons P. A plastic-damage-contact constitutive model for concrete with smoothed evolution functions. Comput Struct. 2016;169:40-56.

72. Gardner D, Jefferson AD, Hoffman A. Investigation of capillary flow in discrete cracks in cementitious materials. Cem Concr Res. 2012;42(7):972-81. https://doi.org/10.1016/j.cemconres.2012.03.017.

73. Gardner D, Jefferson AD, Hoffman A, Lark R. Simulation of the capillary flow of an autonomic healing agent in discrete cracks in cementitious materials. Cem Concr Res. 2014;58:35-44. https://doi.org/10.1016/j.cemco nres.2014.01.005.

74. Gardner D, Herbert D, Jayaprakash M, Jefferson AD, Paul A. Capillary flow characteristics of an autogenic and autonomic healing agent for self-healing concrete. J Mater Civ Eng. 2017;29(11):4017228. https://doi.org/10.1061/(ASCE) MT.1943-5533.0002092.

75. Selvarajoo T, Davies RE, Gardner DR, Freeman BL, Jefferson AD. Characterisation of a vascular self-healing cementitious materials system: flow and curing properties. Constr Build Mater. 2020;245:118332.

76. Jiang T-S, Soo-Gun OH, Slattery JC. Correlation for dynamic contact angle. J Colloid Interface Sci. 1979;69(1):74-7. https://doi.org/10.1016/0021-9797(79)90081-X.

77. Comyn J. Moisture cure of adhesives and sealants. Int J Adhes Adhes. 1998;18(4):247-53. https://doi.org/10.1016/ S0143-7496(97)00031-6.

78. Li YJ, Barthès-Biesel D, Salsac AV. Polymerization kinetics of n-butyl cyanoacrylate glues used for vascular embolization. J Mech Behav Biomed Mater. 2017;69(January):307-17. https://doi.org/10.1016/j.jmbbm.2017.01.003.

79. Alfaiate J, Wells GN, Sluys $\sqcup$ J. On the use of embedded discontinuity elements with crack path continuity for mode-I and mixed-mode fracture. Eng Fract Mech. 2002;69(6):661-86. https://doi.org/10.1016/50013-7944(01)00108-4.

80. Cervera M, Pelà L, Clemente R, Roca P. A crack-tracking technique for localized damage in quasi-brittle materials. Eng Fract Mech. 2010;77:2431-50.

81. Selvarajoo T, Characterisation of a vascular self-healing cementitious material system (PhD thesis), Cardiff University, UK, 2019.

82. Selvarajoo T, Davies RE, Freeman BL, Jefferson AD. Mechanical response of a vascular self-healing cementitious material system under varying loading conditions. Constr Build Mater. 2020;254:119245.

83. Winkler B, Hofstetter G, Niederwanger G. Experimental verification of a constitutive model for concrete cracking Proc Instit Mech Eng Part L J Mater Design Appl. 2001;215(2):75-86.

\section{Publisher's Note}

Springer Nature remains neutral with regard to jurisdictional claims in published maps and institutional affiliations.

\section{Submit your manuscript to a SpringerOpen ${ }^{\odot}$ journal and benefit from:}

- Convenient online submission

Rigorous peer review

- Open access: articles freely available online

- High visibility within the field

Retaining the copyright to your article

Submit your next manuscript at $\boldsymbol{\Delta}$ springeropen.com 